\title{
RESEARCH ADVANCES ON RADIATION TRANSFER MODELING AND INVERSION FOR MULTI-SCALE LAND SURFACE REMOTE SENSING
}

\author{
Qinhuo Liu,
}

State Key Laboratory of Remote Sensing Science, Institute of Remote Sensing applications, Chinese Academy of Sciences

KEYWORDS: Radiation transfer modeling, inversion, multi-scale, multi-source, land surface parameter

\section{ABSTRACT:}

At first, research advances on radiation transfer modeling on multi-scale remote sensing data are presented: after a general overview of remote sensing radiation transfer modeling, several recent research advances are presented, including leaf spectrum model (dPROSPECT), vegetation canopy BRDF models, directional thermal infrared emission models(TRGM, SLEC), rugged mountains area radiation models, and kernel driven models etc.

Then, new methodologies on land surface parameters inversion based on multi-source remote sensing data are proposed. The land surface Albedo, leaf area index, temperature/emissivity, and surface net radiation etc. are taken as examples. A new synthetic land surface parameter quantitative remote sensing product generation system is designed and the software system prototype will be demonstrated.

At last, multi-scale field experiment campaigns, such as the field campaigns in Gansu and Beijing, China will be introduced briefly. The ground based, tower based, and airborne multi-angular measurement system have been built to measure the directional reflectance, emission and scattering characteristics from visible, near infrared, thermal infrared and microwave bands for model validation and calibration. The remote sensing pixel scale "true value" measurement strategy have been designed to gain the ground "true value" of LST, ALBEDO, LAI, soil moisture and ET etc. at 1-km2 for remote sensing product validation. 